\title{
Guía de valoración del paciente crónico en hemodiálisis por indicadores
}

\section{Ana Meizoso Ameneiro - Loreto Sáez Rodríguez - Cristina Valiño Pazos - Sonia Gallego Santiago María Seoane Carro}

Unidad de Hemodiálisis. Complexo Hospitalario Universitario A Coruña. Coruña

\section{Resumen}

La decisión de plantear y diseñar una guía de valoración en nuestra unidad partió de la voluntad y preocupación por compartir el uso de un lenguaje enfermero común y mejorar la calidad de nuestros cuidados, aprovechando la oportunidad que nos ofrece el contar con herramientas como la taxonomía NANDA y la Clasificación de Resultados Enfermeros (NOC).

Elaboramos la "Guía de valoración por indicadores del paciente con insuficiencia renal crónica" planteada como una forma estandarizada de valoración y registro basada en el modelo de patrones funcionales de Marjory Gordon. De la revisión de dichos patrones se seleccionaron los diagnósticos más prevalentes observados en pacientes con insuficiencia renal crónica a tratamiento con hemodiálisis, se relacionaron con los resultados (NOC) esperados después de una intervención enfermera y de forma simultánea los indicadores vinculados al mismo.

Los objetivos a alcanzar incluyen: recoger un conjunto mínimo de datos de manera consensuada, constituir un sistema de valoración integral y fiable, estandarizado y reproducible, establecer la base para identificar problemas reales y potenciales e introducir progresivamente en nuestra práctica clínica un único lenguaje enfermero. Como objetivo global mejoramos la calidad de los registros, incrementando la eficacia y eficiencia de nuestros cuidados y optimizando la comunicación interprofesional.

\begin{tabular}{|c|}
\hline Correspondencia: \\
Ana Meizoso Ameneiro \\
Unidad de Hemodiálisis, $5^{a}$ planta \\
Complexo Hospitalario Universitario A Coruña \\
As Xubias de Arriba $\mathrm{n}^{\circ}: 84$ \\
15.006 A Coruña \\
ameiame@yahoo.es
\end{tabular}

El registro con estas características nos proporciona una visión global y continuada de los cuidados, permite su informatización futura, el análisis comparativo, evita las omisiones o repeticiones, y facilita la observación de los resultados esperados.

PALABRAS CLAVE:

- HEMODIÁLISIS

- GUÍA DE VALORACIÓN

- INDICADOR

- NIC/NOC

- DIAGNÓSTICO ENFERMERO

Guide for the indicator-based evaluation of chronic patients on haemodialysis

\section{Abstract}

The decision to provide and design an evaluation guide in our unit stemmed from the intention and desire to share the use of a common nursing language and improve the quality of our care, taking advantage of the opportunity offered to us by the fact of having tools such as the NANDA taxonomy and the Nursing Outcomes Classification (NOC).

We drew up the "Guide for indicator-based evaluation of patients with chronic renal insufficiency" presented as a standardized form of evaluation and recording based on Marjory Gordon's model of functional patterns. After reviewing these patter$\mathrm{ns}$, the most prevalent diagnoses observed in patients with chronic renal insufficiency undergoing haemodialysis treatment were selected, the expected outcomes (NOC) after a nursing intervention, and at the same time the related indicators, were listed. 
The goals to be attained include: compiling a minimum set of data on a consensual basis, establishing a system of evaluation that is integral and reliable, standardized and reproducible, establishing the basis for identifying real and potential problems and gradually introducing in our clinical practice a single nursing language. As overall goal, we improved the quality of records, increasing the efficacy and efficiency of our care and optimizing interprofessional communication.

Records kept with these characteristics provide us with an overall and continued view of the care, permit future computerization, comparative analysis, avoid omissions and repetitions, and facilitate the observation of the expected outcomes.

\section{KEY WORDS:}

- HAEMODIALYSIS

EVALUATION GUIDE

INDICATOR

NIC/NOC

NURSING DIAGNOSIS

\section{Introducción}

En la era de la revolución tecnológica, la enfermería intenta incorporar sus conocimientos y tareas al campo informático y utilizar la tecnología como instrumento de apoyo y mejora de los cuidados. Para ello resulta imprescindible hablar de un lenguaje común, el idioma de la enfermería del siglo XXI: NANDA-NIC-NOC". "Sin un lenguaje la enfermería es invisible"2 (M. Gordon), éstas son las palabras que han sido fuente de inspiración para comenzar a trabajar en nuestro proyecto: utilizar el lenguaje enfermero estandarizado como herramienta de intercomunicación profesional para mejorar la calidad de nuestros cuidados. Para ello, decidimos utilizar la Taxonomía diagnóstica NANDA combinada con la Clasificación de Resultados Enfermeros NOC como método de registro en la valoración integral de nuestros pacientes. Esta decisión se basó en las posibilidades que ofrece la NOC de proporcionar etiquetas para medir los resultados de las intervenciones enfermeras centrados en el paciente, y de utilizar una escala de medición, de tal forma que podemos registrar los datos no sólo cualitativa sino cuantitativamente.
Con estos criterios hemos elaborado una "Guía de valoración del paciente con IRCT en Hemodiálisis", siguiendo los patrones funcionales de M. Gordon, basada en la valoración de las respuestas humanas desde un enfoque holístico, considerando tanto los problemas como sus efectos sobre la capacidad de la persona para funcionar de modo independiente. Su uso pretende identificar los diagnósticos más prevalentes, relacionándolos con los resultados que esperamos obtener después de una intervención enfermera y utilizando los indicadores como instrumento de medida.

Siguiendo esta metodología de trabajo pretendemos incluir en la práctica diaria el uso de un idioma común, utilizando los lenguajes NANDA y NOC con el fin de mejorar la efectividad de nuestros cuidados. EI progreso, ampliación y desarrollo de nuestro horizonte profesional siempre se proyectará en beneficio del paciente, elevando su calidad de vida.

\section{Objetivos}

- Recoger un conjunto mínimo de datos de manera consensuada

- Constituir un sistema de valoración global y fiable, estandarizado y reproducible

- Establecer la base para identificar problemas y desarrollar un plan de cuidados de máxima efectividad

- Introducir progresivamente en nuestra práctica clínica un único lenguaje enfermero

\section{Material y Método}

$1^{\text {a }}$ Fase: formación. Los miembros del equipo de trabajo realizamos un seminario intensivo de manejo de la nomenclatura NANDA-NIC-NOC, incluido dentro del programa de formación de personal de nuestro complejo hospitalario. Dicha actividad, fue impartida por un experto, constaba de contenido teórico-práctico y tuvo una duración de 40 horas.

$2^{a}$ Fase: búsqueda bibliográfica.

$3^{a}$ Fase: reunión informativa. Dentro del programa semanal de sesiones de enfermería de nuestra uni- 
dad, se incluyó la presentación del proyecto para su discusión.

$4^{\text {a }}$ Fase: valoración del "paciente tipo" a estudio. A través de búsqueda de consenso entre grupo de expertos realizamos una selección entre todos los diagnósticos de enfermería, resultados e indicadores de resultado, aplicables a nuestro paciente tipo, eligiendo aquellos que mostraron mayor prevalencia en el paciente con IRCT en HD. Establecimos la relación NANDA-NOC y determinamos aquellos indicadores estrella más significativos. A través de ellos y según la puntuación otorgada en las escalas tipo Likert detectamos los Diagnósticos Enfermeros (DE) vinculados. De este modo, a partir de la valoración de las respuestas humanas, determinamos los $D E$ presentes en cada individuo.

$5^{\text {a }}$ Fase. Se diseñó el modelo de guía buscando que garantizase las siguientes características: global, fiable, estandarizada, manejable, clara, eficaz y flexible.

Fase final: evaluación de la guía previa implementación. Remitimos la guía para su revisión a varios grupos de expertos: comité de calidad de nuestro complejo hospitalario, personal docente con amplia formación en nomenclatura diagnóstica y personal de enfermería con años de experiencia en el manejo del paciente con IRCT.

Fase actual en desarrollo: integración de la guía de valoración en la unidad. Implementación y retroalimentación evaluativa.

\section{Resultados}

La guía (figura 1) se estructuró en 13 dominios ( $\mathrm{Pa}$ trones de Gordon modificados para su adaptación). En cada dominio se revisaron las diferentes clases con sus correspondientes $D^{3,4}$, y se seleccionaron los diagnósticos más representativos del perfil de usuario con IRCT en HD. De cada diagnóstico se han priorizado los resultados esperados más relevantes, y paralelamente los indicadores más significativos vinculados a estos (figura 2). Seguidamente se otorgó a cada indicador su correspondiente escala de valoración tipo Likert, de cinco puntos que cuantificó en un continuo, desde el menos al más deseable. Según la puntuación asignada al indicador en el proceso de valoración, se logró confirmar o descartar cada diagnóstico. Este formato permite, a través de la medición de los indicadores, la selección de los DE vinculados a cada resultado, obteniendo un plan de cuidados individualizado de forma simultánea a la valoración.

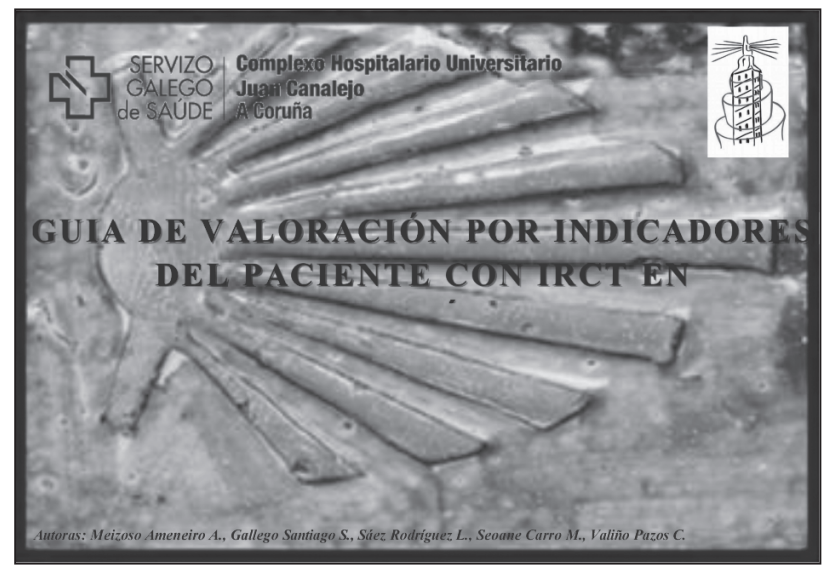

Figura 1. Guía de valoración del paciente en hemodiálisis

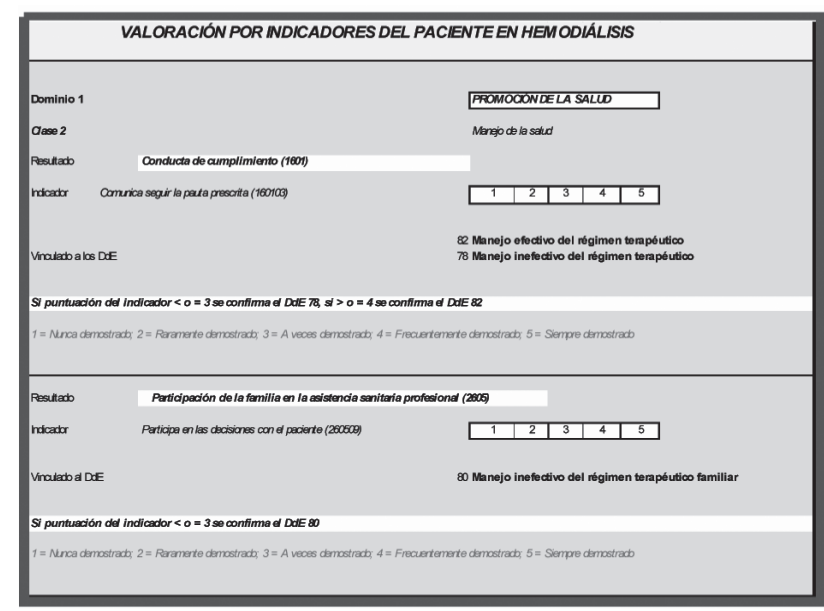

Figura 2. Valoración por indicadores

A través del diseño de esta guía, cumplimos los objetivos planteados como meta al inicio de este proyecto: tenemos un instrumento de valoración estandarizado, que se puede reproducir, que permite una recogida de datos consensuada y que nos ayuda en la identificación de problemas.

Por otro lado, descubrimos una nueva utilidad de la guía que da solución a un objetivo no planteado al inicio de nuestro trabajo pero que pensamos es importante reflejar en los resultados del mismo. Con nuestro proyecto, hemos conseguido aumentar el interés y motivación del personal de enfermería de 
nuestra unidad hacia los diagnósticos de enfermería y el registro a través de taxonomías diagnósticas. La decisión de incluir en la guía un anexo que clarificase la terminología empleada (anexol) como ayuda a enfermeras noveles en este campo, se reveló como un gran acierto y despertó la curiosidad de profesionales que hasta entonces permanecían alejados e incluso escépticos ante las tendencias unificadoras del lenguaje enfermero. La obtención de este resultado inesperado constituye un gran refuerzo positivo para el equipo investigador.

\section{Discusión}

Esta guía facilita la incorporación del lenguaje enfermero a la práctica enfermera, mejorando la calidad de los registros y permitiendo que el personal de enfermería sin conocimientos previos de la taxonomía, identifique las etiquetas diagnósticas presentes en sus pacientes. Además, como instrumento validado y universal, resulta extrapolable a la dinámica de registro de otros centros, siendo flexible para su adaptación a cada caso particular.
En el contexto de la etapa de cambio que nos ha tocado vivir y con el objetivo de mejorar de forma unánime la calidad de los registros, pensamos que la valoración por indicadores permite establecer de forma paralela los diagnósticos de enfermería y los resultados que esperamos. A través de la medición de los indicadores queda reflejada una valoración cuantitativa, de forma que se objetiva, con la evolución en el tiempo, la efectividad de nuestras intervenciones.

Por otro lado, esta guía incluye el dominio 13 "Crecimiento y desarrollo", de gran relevancia debido al aumento progresivo de población geriátrica. Se recoge así el diagnóstico de enfermería Incapacidad del adulto para mantener su desarrollo, presente en un alto porcentaje de pacientes que acuden a hemodiálisis. Los indicadores vinculados a éste DE son: memoria, agudeza visual, agudeza auditiva y capacidad cognitiva, correspondientes a su vez al resultado de Envejecimiento físico. Este dominio no ha sido contemplado por otros autores en anteriores Guías ${ }^{5}$, por lo que aportamos un punto de innovación con una herramienta que se suma a las ya existentes, para facilitar el manejo de las Taxonomías NANDA-NOC.

Resultado: Un estado, conducta o percepción de un individuo, familia o comunidad medida a lo largo de un continuo en respuesta a intervención(es) de enfermería. Cada resultado tiene asociado un grupo de indicadores que son utilizados para determinar el estado del paciente en relación al resultado. Para medirlo, el resultado precisa la identificación de una serie de indicadores más específicos.

Indicador de un resultado: Un estado, conducta o percepción más concreto de un individuo, familia o comunidad que sirve como indicación para medir un resultado. Los indicadores de los resultados del paciente susceptibles a las intervenciones enfermeras caracterizan el estado de un paciente, familia o comunidad a nivel concreto.

Medición: Escala tipo Likert de cinco puntos que cuantifica el estado del resultado o indicador de un paciente en un continuo, desde el menos al más deseable, y proporciona una puntuación en un momento dado. La medición reflejará un continuo, como por ejemplo $1=$ gravemente comprometido; 2 = sustancialmente comprometido; 3 = moderadamente comprometido; 4 = ligeramente comprometido; $5=$ no comprometido.

Taxonomía NOC: Organización sistemática de resultados en grupos o categorías basadas en semejanzas, diferencias y relaciones entre los resultados. La estructura de la taxonomía de la NOC tiene cinco niveles: dominios, clases, resultados, indicadores y medidas.

Diagnóstico enfermero (DE): Juicio clínico sobre la respuesta de una persona, familia o comunidad frente a procesos vitales/ problemas de salud reales o potenciales. El diagnóstico enfermero proporciona la base para la selección de las intervenciones enfermeras destinadas a lograr los objetivos de los que la enfermera es responsable (aprobado en la novena conferencia, 1990).

Etiqueta del diagnóstico: Proporciona un nombre al diagnóstico. Es un término o frase concisa que representa un patrón de claves relacionadas.

Taxonomía II: Tiene tres niveles: dominios, clases y diagnósticos enfermeros. La Taxonomía II presenta: 13 dominios, 47 clases y 188 diagnósticos.

Dominio: Un dominio es una "esfera de actividad, estudio o interés" (Roget, 1980, pág. 287)

Clase: Una clase es "una subdivisión de un grupo mayor; una división de las personas o casas por su calidad, rango o grado" (Roget, 1980; pág 157)

Nomenclatura: Sistema de designaciones (términos) elaborado según unas reglas preestablecidas (American Nurses Association, 1999)

Taxonomía: Clasificación según las relaciones naturales que se suponen entre los tipos y subtipos (American Nurses Association, 1999).

Interrelación: Aquello que dirige una asociación de conceptos.

Anexo 1. Glosario de términos utilizados en la guía 
Una herramienta útil para el proceso de atención de enfermería, una guía que nos dirige hacia un plan de cuidados enfermeros monitorizado, eficaz y eficiente, hacia una enfermería basada en la evidencia y que camina hacia la excelencia. Hemos dado el primer paso para que nuestra unidad de diálisis abandone los dialectos y hable un único idioma oficial: el lenguaje enfermero NANDA-NIC-NOC.

\section{Bibliografía}

1. Johnson M, Bulechek G, Butcher H, McCloskey Dochterman J, Maas M, Morhead S, Swanson E. Interrelaciones NANDA, NOC y NIC. $2^{\mathrm{a}}$ ed. Madrid: Elsevier; 2007.

2. Alfaro-Le Fevre R. Aplicación del proceso enfermero. Fomentar el cuidado en colaboración. $5^{a}$ ed. Barcelona: Masson; 2003.
3. Morhead S, Johnson M, Maas M. Clasificación de Resultados de Enfermería (NOC). Madrid: Elsevier; 2005.

4. NANDA International. Diagnósticos enfermeros. Definiciones y Clasificación (2007-2008). Madrid: Elsevier; 2007.

5. Salces Sáez E, del Campo Romero MC, Carmona Valiente MC, Fernández García JL, Fernández Montero JJ, Ramírez Rodríguez A. Guía de Diagnósticos Enfermeros del paciente en hemodiálisis (Interrelaciones NANDA-NOC-NIC). Madrid: 2005. 